\title{
Improve Thermal Conductivity of Polymer Composites via Conductive Network
}

\author{
Lilin $\mathrm{He}$
}

Received date: 19 February 2021; Accepted date: 15 April 2021.

Article type: Editorial article.

While the past decades have witnessed the exponential increase of power and energy densities in electronic devices, the efficient thermal management is critical for the performance, reliability, lifetime of these devices. Polymers have advantages over metals and other materials for heat dissipation in the electronics due to their desirable properties, such as low cost, light weight, flexibility, chemical stability, excellent electrical insulation and processability. However, polymer-based materials usually exhibit very low thermal conductivity in the range of $0.1-0.5 \mathrm{~W} \mathrm{~m}^{-1} \mathrm{~K}^{-1}$, which is attributed to the declined phonon's mean free path caused by amorphous domains in polymers, i.e. chain dangling ends, chain misalignment, entanglement, loop, random orientation, voids and impurities etc..$^{[1,2]}$

There have been enormous efforts devoted into the enhancements of the thermal conductivity of polymeric materials. ${ }^{[3-5]}$ One of the strategies is to improve the alignment of polymer chains by mechanical stretching, electrospinning, nanoscale templating and external fields. Another strategy is to engineer the polymer chain structures via rational design of the backbone bonds, side chains, covalent cross-link, and intra-chain interactions. In addition to these efforts, it is also promising to incorporate thermally conducting fillers such as carbon derivatives, ceramics and metallic into polymer matrix for the applications where efficient heat dissipation is needed. The final conductivity of the composites is determined by not only the concentration, size, shape and conductivity of the fillers, but also the interactions between the fillers and polymer chains.

While important progresses have been made in the past years, there are still many challenges in the enhancement of the thermal conductivity in polymer composites. The new electronic needs low loading of conductive fillers for high thermal conductivity because the high loading increases the cost and compromises the mechanical properties, flexibility

Neutron Scattering Division, Oak Ridge National Laboratory, Oak

Ridge, Tennessee 37831, United States

Email: hel3@ornl.gov (L. He) and processibility. Another challenge is the aggregation of fillers and large inter-filler thermal contact resistance. Therefore, Novel approaches to process the materials are needed to further improve the thermal conductivity of polymeric materials.

Modelling methods play an essential role in the understanding of how different factors influence the thermal conductivity of polymer composites, which further guides the design of the novel materials. ${ }^{[6,7]}$ Classical models for composites are categorized as two major groups: effective medium approximation and the micromechanics methods. Unfortunately, none of these models succeed in accurately describing the thermal conductivity behaviors of composite materials due to the complicated structure and composition. Among these models, Nan model considering the effects of volume fraction of fillers, interfacial thermal resistance, particle size and aspect ratio, and orientation distribution has gained increasing attention. Writing in Engineered Science Materials, Sun J. et al. proposed a new method to evaluate the thermal conductivity enhancement via thermal conductive network (TCN) ${ }^{[8]}$ They also designed a model system with conductive polydimethylsiloxane (PDMS), glass bubble (GB) and short carbon fiber (SCF) to test their model. The good agreement between calculated results with the model and the experimental data indicates the model is promising.

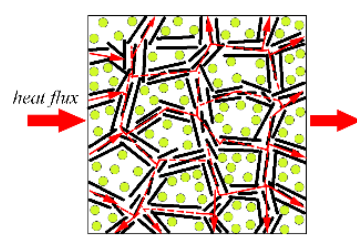

(a)

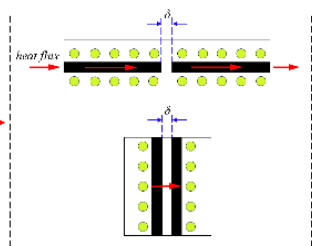

(b)

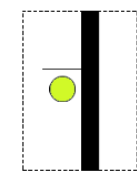

(c)
- Carbonfiber $\bigcirc$ (ilass bubble $\sqsupset$ Polymer $\quad \delta:$ Polymer layer between carbon fibers

Fig. 1 (a) Proposed TCN structure in the polymer composites with the hybrid fillers; (b)Two types of heat flow pathways; (c) The unit cell that is simplified for the calculation in the proposed model. (Reproduced with the Permission form [8], Copyright 2021 Engineered Science Publisher LLC) 
In the new model proposed by Sun J. et al., two techniques, TCN densification via the spatial confining forced network assembly (SCFNA) and rigid particles were combined. ${ }^{[8]}$ They improved the model based on the Nan model. Subsequently they synthesized PDMS composites using glass bubble and short carbon fiber as a model system to validate their theory. The effects of volume fraction, thermal boundary resistance, thermal contact resistance, aspect ratio and distribution state of fillers on the thermal conductivity of this composite system were studies and compared with the calculated results. The comparison showed good agreement between the calculated results and experimental data. This improved model sheds new insights into the TC enhancement of polymer composites.

\section{References}

[1] C. Huang, X. Qian, R. Yang, Mat. Sci. Eng. R, 2018, 132, 122, doi: 10.1016/j.mser.2018.06.002.

[2] L. Zhang, H. Deng, Q. Fu, Compos. Commun., 2018, 8, 74 82, doi: 10.1016/j.coco.2017.11.004.

[3] V. Singh, T. L. Bougher, A. Weathers, Y. Cai, K. Bi, M. T. Pettes, S.A. McMenamin, W. Lv, D. P. Resler, R.R. Gattuso, D. H. Altman, K. H. Sandhage, L. Shi, A. Henry, B. A. Cola, Nat. Nanotechnol., 2014, 9, 384-390, doi: 10.1038/nnano.2014.44.

[4] S. N. Leung, Compos. Part B, 2018, 150, 78-92, doi: 10.1016/j.compositesb.2018.05.056.

[5] A. Tessema, D. Zhao, j. Moll, S. Xu, R. Yang, C. Li, S. K. Kumar, A. Kidane, Polym. Test., 2017, 57, 101-106, doi: 10.1016/j.polymertesting.2016.11.015.

[6] A. Agrawal, A. Satapathy, Int. J. Therm. Sci., 2015, 89, 203209, doi: 10.1016/j.ijthermalsci.2014.11.006.

[7] S. Zhai, P. Zhang, Y. Xian, J. Zeng, B. Shi, Int. J. Heat Mass Transf., 2018, 117, 358-374, doi: 10.1016/j.ijheatmasstransfer.2017.09.067.

[8] J. Sun, X. Zhang, Q, Du, V. Murugadoss, D. Wu, Z. Guo, ES Mater. Manuf., 2021, 13, doi: 10.30919/esmm5f450.

Publisher's Note: Engineered Science Publisher remains neutral with regard to jurisdictional claims in published maps and institutional affiliations. 